NASA Technical Memorandum 88219

NASA-TM-88219 19860019258

\title{
A Simple Antireflection Overcoat for Opaque Coatings in the Submillimeter Region
}

Sheldon M. Smith

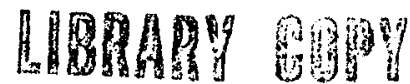

MAYO BOBS

LANGLEY RESEARCH CENTER

LIBRARY, NASA

MAMTON, VIRGINIA

\section{N/SA}

National Aeronautics and

Space Administration 
NASA Technical Memorandum 88219

\section{A Simple Antireflection Overcoat for Opaque Coatings in the Submillimeter Region}

Sheldon M. Smith, Ames Research Center, Moffett Field, California

April 1986

\section{N/SN}

National Aeronautics and

Space Administration

Ames Research Center

Moffett Field, California 94035 
A simple antireflection overcoat for opaque coatings in the submillimeter region

Sheldon M. Smith

NASA Ames Research Center, Moffett Field, CA 94035

Abstract

An antireflection overcoat for opaque baffle coatings in the FIR/submillimeter region has been made from a simple Teflon spray-on lubricant. The Tefion overcoat reduced the specular reflectance of four different opaque coatings by nearly a factor of two. Analysis, based on the interference term of a reflecting-layer model, indicates that in the submillimeter region the reduced reflectance depends primarily on the refractive index of the overcoat and very little on its thickness.

I. Introduction

As 1llustrated by an earlier paper ${ }^{1}$ (hereafter called Paper I), the need for low reflectance baffles inside far-infrared (FIR) space telescopes such as SIRTF (the Space Infrared Telescope Facility) has stimulated a survey of FIR absorbing materials. Another way to achieve low reflectance is by means of an antireflection coating. The technique for doing this in transmission with smooth, nonabsorbing substrates is wellknown, ${ }^{2}$ however the method used in reflection with smooth, absorbing coatings is not as well known. The antireflection technique used in reflection has been referred to by the terms gradual-transition absorber, ${ }^{3}$ multilayer absorber, ${ }^{4}$ and multilayer coating. 5 The purpose 
of such a coating is to match the optical constants of free space, which are relatively small, with those of the substrate, which are very large. A similar problem occurs in acoustics. At optical wavelengths Heavens ${ }^{6}$ suggested a film whose refractive index varied continuously from one medium to the other, and Jacobsson and Martensson ${ }^{7}$ have actually succeeded in making such a graded-index film. In the microwave region the wavelength is long enough that layers of material can be used to approximate a continuous transition. Thus in a multilayer coating the refractive index increases irregularly from the outside inward (hence the surface reflectance of the outermost layer is the least). This paper describes a simple, single-layer antireflection overcoat for rough, high-index, baffle coatings.

Both polyethylene and mylar have been used to make FIR antireflection coatings 8 in transmission. Teflon (polytetrafluoroethylene) is somewhat similar chemically, but with a lower index of refraction. Because of its low index, four IR-opaque baffle coatings described in Paper I were sprayed with Teflon to see whether it would have an antireflection effect in reflection. The material used was Teflon Wet Lubricant (the Du Pont Co., Wilmington, DE 19898), and a "heavy" spray was applied in an effort to produce a thick layer. Specular reflectance spectra (as described in Paper I) were made of the four coatings both before and after they were sprayed so that any changes measured would be attributable to the Teflon overcoat.

The analysis which follows uses the Teflon refractive index obtained by Chamberlain and $\mathrm{Gebbie}^{9}$ because their value is quite close to that obtained by Alvarez, Jennings, and Moorwood ${ }^{10}$ after a $5.5 \%$ temperature shift observed in the latter's polyethylene data was applied 
to their Teflon data. The value found by Birch et al. ${ }^{11}$ is only. $3.5 \%$ higher. Beyond $65 \mu \mathrm{m}$, Chamberlain and Gebbie show that the absorption coefficient of Teflon is negligible. The optical constants of the four combination coatings were estimated in Paper I and they will be used here (Table I) with the comment that they are sufficiently accurate for a first-order analysis.

\section{Observations}

Figures 1, 2, 3, and 4 present reflectance spectra made near normal incidence of four combination coatings both before and after they were sprayed with Teflon Wet Lubricant. The thickness, d, and roughness, $\sigma$, of each coating are given in parenthesis $(d, \sigma)$ in the figure captions together with the sample number and coating description. In Fig. 1, the short wavelength data have been smoothed during replotting at the scale of the longer wavelength data. The reflectance levels shown in Figs. 2, 3 , and 4 are considerably below those in Fig. 1 because the coatings in the latter figures are much thicker. For that reason, and also because a thinner beam splitter was used, the short wavelength spectra of Fig. 4 are noisy; however, they still show two clearly separated reflectance levels beyond $80 \mu \mathrm{m}$.

Two inajor effects of the Teflon overcoat are apparent in the strong spectra of Fig. 1. First, the peak at $77 \mu \mathrm{m}$ is shifted $(8 \mu \mathrm{m})$ toward longer wavelength, and second, the reflectance at wavelengths beyond $300 \mu \mathrm{m}$ is reduced by a factor close to two. In Fig. 2 both the reduced reflectance and the wavelength shift occur at the same feature. The spectra in Figs. 3 and 4 are of quite rough coatings and they demonstrate significantly reduced reflectance in two different regions of the 
spectrum. To summarize, these observations demonstrate an approximately $50 \%$ reduction of the reflectance of each sample tested, and two samples show the shift of a spectral feature to longer wavelength.

\section{Analysis}

The two effects demonstrated in the preceding section appear large enough and simple enough to be amenable to a first-order analysis. $\mathrm{Smith}^{12}$ has shown that as the wavelength increases from 10 to about $350 \mu \mathrm{m}$ the reflectance of many optical-black coatings increases rapidly because the coating's exponential absorption coefficient $(4 \pi k d / \lambda)$ decreases almost linearly. Concurrently, interference between the specular reflections from the upper (coating) and lower (metal) surfaces produces characteristic fringes which have been called "channelled" spectra. ${ }^{13}$ The peaks in Figs. 1 and 2 are examples of such fringes. In the absence of the Teflon overcoat indicated in Fig. 5, the unshifted peaks are due to constructive interference largely between beams $B$ and $C$ of the figure. (Multiple internal reflections are rapidly attenuated in such thick, absorbing coatings.) When an overcoat is present as shown, the shifted peaks are then due to interference largely between beams $A$ and $\mathrm{C}$.

The simple analysis just described is suggested by two observations: first, the shift to longer wavelength of interference features is consistent with a larger path difference created by the overcoat; and second, the changes observed are only simple modifications of the original interference pattern which indicates that just two beams are still involved. If an additional beam were significantly involved, additional features such as shoulders, plateaus, or other peaks would be 
apparent. Thus it will be assumed that after an overcoat has been applied, the principal interference interaction occurs between beams $A$ and $C$ and any further interaction with beam $B$ can be neglected in $a$ first-order analysis.

Because interference features apparently dominate the FIR spectra of these rough coatings, the interference term of the reflecting-layer model can be used to evaluate them. The second term of Eq. (18) of Ref. 12 gives

$R_{\text {interference }}=2 \cos \left(\frac{4 \pi n d}{\lambda}\right)\left[R_{1} \exp -\left(\frac{4 \pi \sigma_{1}}{\lambda}\right)^{2}\right]^{1 / 2} T_{\text {spec }} \exp \left(\frac{-4 \pi k d}{\lambda}\right)$,

where $n \pm i k$ is the complex refractive index of the coating, $d$ is its thickness, $\lambda$ is the wavelength, $\sigma_{1}$ is the rms roughness of the upper surface, and $R_{1}$ is its Fresnel reflectance as given by the usual formula,

$$
R_{1}=\frac{(n-1)^{2}+k^{2}}{(n+1)^{2}+k^{2}}
$$

In Eq. (1B) of Ref. 12, we have set $R_{2}=1$ and $\sigma_{2}=0$ for a smooth metal substrate, and $n_{0}=1$ for air (see Fig. 5). The presence of interference fringes indicates that the wavelength is sufficiently long that diffuse transmission through the upper surface can also be neglected, hence

$$
\mathrm{T}_{\text {spec }} \simeq 1-\mathrm{R}_{1}
$$


Figure 5 shows the situation when the basic coating has been covered with a nonabsorbing overcoat of thickness $d_{f}$ and (real) refractive index $n_{f}$. For simplicity, it is assumed that the overcoat does not change the roughness, $\sigma_{1}$.

The first-order effect of a nonabsorbing overcoat on the normal incidence specular reflectance can be calculated from the ratio formed by Eq. (1) evaluated with an overcoat (beams $A$ and $C$ ), divided by Eq. (1) evaluated without the overcoat (beams B and C). The evaluation involves substituting a new optical path, nd. $\sec (\theta / n)+n_{f} d_{f} \cdot \sec \left(\theta / n_{f}\right)$, for the original path $n d \cdot \sec (\theta / n)$ in the cosine factor, inserting the appropriate refractive index throughout, and selecting the appropriate wavelength. The $\sec (\theta / n)$ factors provide small corrections for notquite normal incidence.

In the ratio so formed different factors cancel depending on whether the evaluation is done at the peak of an interference fringe, which will specify different wavelengths, or whether it is done at an arbitrarily selected single wavelength. In the former case, called Technique I, phase is constant and the cosine factors cancel leaving

Tech I :

$$
\frac{R_{W i}}{R_{W O}}=\frac{R_{1 A}^{1 / 2}\left(1-R_{1 A}\right)}{R_{1 B}^{1 / 2}\left(1-R_{1 B}\right)}\left[\frac{\exp -\left(\frac{4 \pi \sigma_{1}}{\lambda_{A}}\right)^{2}}{\exp -\left(\frac{4 \pi \sigma_{1}}{\lambda_{B}}\right)^{2}}\right]^{1 / 2} \frac{\exp \left(\frac{-4 \pi k d \sec (\theta / n)}{\lambda_{A}}\right)}{\exp \left(\frac{-4 \pi k d \sec (\theta / n)}{\lambda_{B}}\right)},
$$

where $R_{1 B}$ is the $R_{1}$ of Eq. (2), and

$$
R_{1 A}=\frac{\left(n_{f}-1\right)^{2}}{\left(n_{f}+1\right)^{2}}
$$


The peak wavelengths are

$$
\lambda_{A}=\lambda_{B}+\Delta \lambda \text {, }
$$

where $\Delta \lambda$ is measured on Figs. 1 and 2. Note that Eq. (4) is independent of $d_{f}$, the thickness of the overcoat. In the latter case, called Technique II, the exponential factors cancel because $\Delta \lambda$ is zero and Teflon is nonabsorbing. Thus

Tech II:

$$
\frac{R_{W i}}{R_{W o}}=\frac{R_{1 A}^{1 / 2}\left(1-R_{1 A}\right)}{R_{1 B}^{1 / 2}\left(1-R_{1 B}\right)} \frac{\cos \left[\frac{4 \pi}{\lambda}\left\{n d \sec (\theta / n)+n_{f} d_{f} \sec \left(\theta / n_{f}\right)\right\}\right]}{\cos \frac{4 \pi n d \sec (\theta / n)}{\lambda}} .
$$

By equating phase factors at the interference peaks, the optical thickness of the overcoat can be related to the wavelength shift, yielding

$$
\frac{n_{f} d_{f} \sec \left(\theta / n_{f}\right)}{n d \sec (\theta / n)}=\frac{\Delta \lambda}{\lambda_{B}}
$$

Substituting Eq. (8) into (7) produces a second equation also independent of the overcoat thickness:

$$
\frac{R_{w i}^{\prime}}{R_{w O}}=\frac{R_{1 A}^{1 / 2}\left(1-R_{1 A}\right) \cos \left[\frac{4 \pi d}{\lambda}\left\{n \sec (\theta / n)+n^{\prime} \sec \left(\theta / n^{\prime}\right) \frac{\Delta \lambda}{\lambda_{B}}\right\}\right]}{R_{1 B}^{1 / 2}\left(1-R_{1 B}\right) \cos \frac{4 \pi n d \sec (\theta / n)}{\lambda}}
$$

where $n^{\prime}$ is the value of $n$ measured at $\lambda_{B}$.

Examination of Eqs. (4), (7), and (9) indicates that as the wavelength becomes large, the only factors that do not explicitly approach 
unity are those involving the surface reflectance coefficients, $R_{1 A}$ and $R_{1 B}$. Since these coefficients depend only on optical constants which in the submillimeter region change quite slowly, it appears that the submillimeter reflectance ratio approaches a nearly constant value given by

$$
\frac{R_{W I}}{R_{W O}}=\frac{R_{1 A}^{1 / 2}\left(1-R_{1 A}\right)}{R_{1 B}^{1 / 2}\left(1-R_{1 B}\right)} \text {, }
$$

which is independent of the thickness of the overcoat. Even at shorter wavelengths, one would still expect the reduced reflectance to be strongly governed by this value. Substitution of Eqs. (2) and (5), followed by some algebraic manipulation yields

$$
\frac{R_{w i}}{R_{w o}} \simeq \frac{n_{f}\left(n_{f}-1\right)\left[(n+1)^{2}+k^{2}\right]^{3 / 2}}{\left(n_{f}+1\right)^{3} n\left[(n-1)^{2}+k^{2}\right]^{3 / 2}} .
$$

This complicated relation has a nonlinear dependence on the optical constants, but for the expected values of $n \geq 1$ and $k \geq 0.3$, its dependence on $\mathrm{n}$ and $\mathrm{k}$ is small. The numerator, however, has a regular dependence on $n_{f}$ and it goes to zero as $n_{f}$ decreases to 1 . Thus the most significant parameter of the reduced reflectance in the submillimeter region appears to be the index of refraction of the overcoat, and not its thickness. Contrary to what might be expected from analogy to antireflection coatings in transmission, the reflectance in this case decreases regularly with decreasing overcoat index. 


\section{Calculations}

In this section, Eqs. (4), (7), (8), and (9) are applied. For these calculations the roughness and thickness of the IR-opaque coatings are taken from Paper I together with the estimates of their optical constants. The dispersion of Teflon is so small in the FIR that the value of the refractive index given by Chamberlain and Gebbie $9,1.384$, is essentially independent of wavelength. The angle of incidence on the coating was $17^{\circ}$ and the significant wavelengths are indicated in Figs. 1 and 2. The ratios Rwi/Rwo were measured in Figs. 1 through 4 . These data are listed in Table I together with the results of the calculations.

The first two calculations apply Eq. (4) to the spectral peaks shown in Figs. 1 and 2. Considering the approximations made and the estimated nature of the optical constants of the coatings, the calculations show good agreement with measurement. The large differences' in applied wavelength, in the measured reflectances and reflectance ratios, and in coating thicknesses between the two applications indicate that Technique $I$ is valid over a wide range of FIR wavelengths. It was also evident during the calculations that the ratio of reflectance coefficients, $R_{1 A}$ and $R_{1 B}$, contributed more to the final result than did the other factors, as suggested by Eq. (10).

The third calculation applies Eq. (9) to sample $\# 45$ at $400 \mu \mathrm{m}$ by utilizing the wavelength shift of the short wavelength feature at $77 \mu \mathrm{m}$. This process required an evaluation of the optical constants of the combination coating at two widely separate wavelengths. Considering this, the difference of $23 \%$ between calculation and measurement is not considered poor. 
In the absence of a spectral feature, Technique II can be applied if the thickness of the overcoat is known. Considering the success of the first two calculations, the thickness of the overcoat on those two coatings was calculated by Eq. (8). The results gave $4.3 \mu \mathrm{m}$ on $\# 45$ and $24 \mu \mathrm{m}$ on $\$ 32$. In view of the absence of any control on the thickness of the overcoat, this large spread in values is not too surprising. A simple average of these two values was then taken to obtain a representative value $(15 \mu \mathrm{m})$ of overcoat thickness to use in applying Eq. (7) to samples $\# 30$ and $\# 25$. It should be noted here that these two combination coatings are not homogeneous (the SiC particles are isolated facets) hence their estimated optical constants are more uncertain than those of the previous samples. Using the value of $15 \mu \mathrm{m}$ for $d_{f}$, Eq. (7) calculates a reflectance ratio for sample $\# 30$ at $400 \mu \mathrm{m}$ that is only $26 \%$ different from the value measured on Fig. 3.

Finally, with $d_{f}$ initially equal to $15 \mu \mathrm{m}$, Eq. (7) was applied to sample \#25 (Fig. 4) at $100 \mu \mathrm{m}$. It was quickly found that with such a thick coating at short wavelength that the cosine factors oscillate so fast the calculated ratio can take on any of a large range of values depending on the specific values of $d, d_{f}$, or $\lambda$. This result is supported by the long wave half of Fig. 4 which has much more the appearance of channelled spectra than of noise. Although this first-order analysis breaks down in this case, it should be noted that the calculated ratio based on just the reflectance coefficients (Eq. (10)) is of the correct order, 0.66 , when compared to the value of 0.5 obtained from smooth curves estimated through the spectra of Fig. 4. 
V. Summary and Conclusions

A simple antireflection overcoat for FIR-opaque baffle coatings has been made by spraying the coatings with Teflon Wet Lubricant. The specular reflectance of four different coatings was reduced by approximately a factor of two in the F'IR/submillimeter region. A first-order aralysis based on the interference term of a reflecting-layer model was used to calculate the reduced reflectance to within $26 \%$. This analysis indicates that in the submillimeter region the reduction in specular reflectance depends primarily on the refractive index of the overcoat and very little on its thickness. 


\section{References}

1. S. M. Smith, "Survey of Material for an Infrared-Opaque Coating," to be published as NASA TM-88204, Dec. 1985.

2. M. Born and E. Wolf, Principles of Optics, (Pergamon Press, Oxford, 1970) 4th Ed., p. 64.

3. K. Walther, "Reflection Factor of Gradual Transition Absorbers for Electron and Acoustic Waves," I.R.E., AP-8, 608-621 (1960).

4. A. R. Jones and E. R. Wooding, "Multilayer Microwave Absorber," IEEE, AP-12, 508-509 (1964).

5. J. R. Grammer, L. J. Bailin, M. D. Blue, and S. Perkowitz, "Absorbing Coatings for the Far Infrared," Proc. Soc. Photo-Opt. Instrum. Eng., 257, 192-195 (1980).

6. 0. S. Heavens, Optical Properties of Solid Thin Films (University Microfilms, Ann Arbor, Mich., 1964), p. 208.

7. R. Jacobsson and J. O. Martensson, "Evaporated Inhomogeneous Thin Films," Appl. Opt., $\underline{5}, 29$ (1966).

8. K. R. Armstrong and F. J. Low, "FIR Filters Utilizing Small Particle Scattering and Anti-Reflection Coatings," Appl. Opt., 13, 427 (1974).

9. J. E. Chamberlain and H. A. Gebbie, "Dispersion Measurements on Polytetrafluoroethylene in the Far Infrared," Appl. Opt., $\underline{5}, 393$ (1966).

10. J. A. Alvarez, R. E. Jennings, and A. F. M. Moorwood, "IR Measurements of Selected Optical Materials at $1.6^{\circ} \mathrm{K}$," Infrared Phys., 15, 45 (1975). 
11. J. R. Birch, J. D. Dromey, and J. Lesurf, "Optical Constants of Some Low-Loss Polymers between 4 and $40 \mathrm{~cm}^{-1}$," Infrared Phys., 21, 225 (1981).

12. S. M. Smith, "Specular Reflectance of Optical-Black Coatings in the Far Infrared," Appl. Opt., 23, 2311-2326 (1984).

13. R. W. Ditchburn, Light (Blackie and Son, Glasgow, 1953), p. 141. 
Table I. Comparison of Calculations with Measurements of the Reflectance Ratio

\begin{tabular}{|c|c|c|c|c|c|c|c|c|c|c|}
\hline \multirow{2}{*}{$\begin{array}{l}\text { Sample } \\
\text { number }\end{array}$} & \multirow{2}{*}{$\begin{array}{c}\text { Combination } \\
\text { coating }\end{array}$} & \multirow{2}{*}{$\begin{array}{l}\text { Tech- } \\
\text { nique }\end{array}$} & \multirow[b]{2}{*}{$d_{(\mu m)}$} & \multirow[b]{2}{*}{$\sigma_{1}(\mu \mathrm{m})$} & \multirow[b]{2}{*}{$\lambda_{B}(\mu \mathrm{m})$} & \multirow[b]{2}{*}{$\Delta \lambda / \lambda$} & \multirow[b]{2}{*}{$n / k$} & \multicolumn{3}{|c|}{ Rwi/Rwo } \\
\hline & & & & & & & & $\begin{array}{l}\text { Calu- } \\
\text { lated }\end{array}$ & $\begin{array}{l}\text { Mea- } \\
\text { sured }\end{array}$ & $\begin{array}{l}\text { Difference } \\
\text { from } \\
\text { measurement }\end{array}$ \\
\hline 45 & $\mathrm{ECP} / \mathrm{CB} / \mathrm{TI} \mathrm{Br}$ & I & 40 & 3 & 77 & 0.104 & $1.45 / 0.3$ & 0.93 & 1.0 & $-7 \%$ \\
\hline 32 & Chemg/CB/TlBr & I & 210 & 7.5 & 357 & 0.048 & $3.50 / 1.1$ & 0.59 & 0.53 & $+11 \%$ \\
\hline 45 & $\mathrm{ECP} / \mathrm{CB} / \mathrm{T} 1 \mathrm{Br}$ & II & 40 & 3 & 400 & 0.104 & $2.90 / 1.1$ & 0.36 & 0.47 & $-23 \%$ \\
\hline 30 & Chemg/CB/SiC & II & 160 & 30 & 400 & $\left(d_{f}=15 \mu m\right)$ & $2.95 / 1.1$ & 0.58 & 0.46 & $+26 \%$ \\
\hline 25 & $\mathrm{ECP} / \mathrm{CB} / \mathrm{SiC}$ & II & 215 & 25 & 100 & $\left(d_{f}=15 \mu m\right)$ & $1.35 / 0.5$ & -0.66 & -0.5 & $-\infty$ \\
\hline
\end{tabular}




\section{Figure Captions}

Fig. 1. Sample 45: TIBr and carbon black in ECP-2200, $(40,3)$.

Fig. 2. Sample 32: TIBr and carbon black in Chemglaze 2-306, $\left(2.10,7 \frac{1}{2}\right)$.

Fi.g. 3. Sample 30: Number $80 \mathrm{SiC}$ grit and carbon black in Chemglaze $2-306,(160,30)$.

Fig. 4. Sample 25: Number $80 \mathrm{SiC}$ grit and carbon black in ECP-2200, $(215,25)$.

Fig. 5. Schematic view of overcoat of a combination coating on a reflecting substrate. 


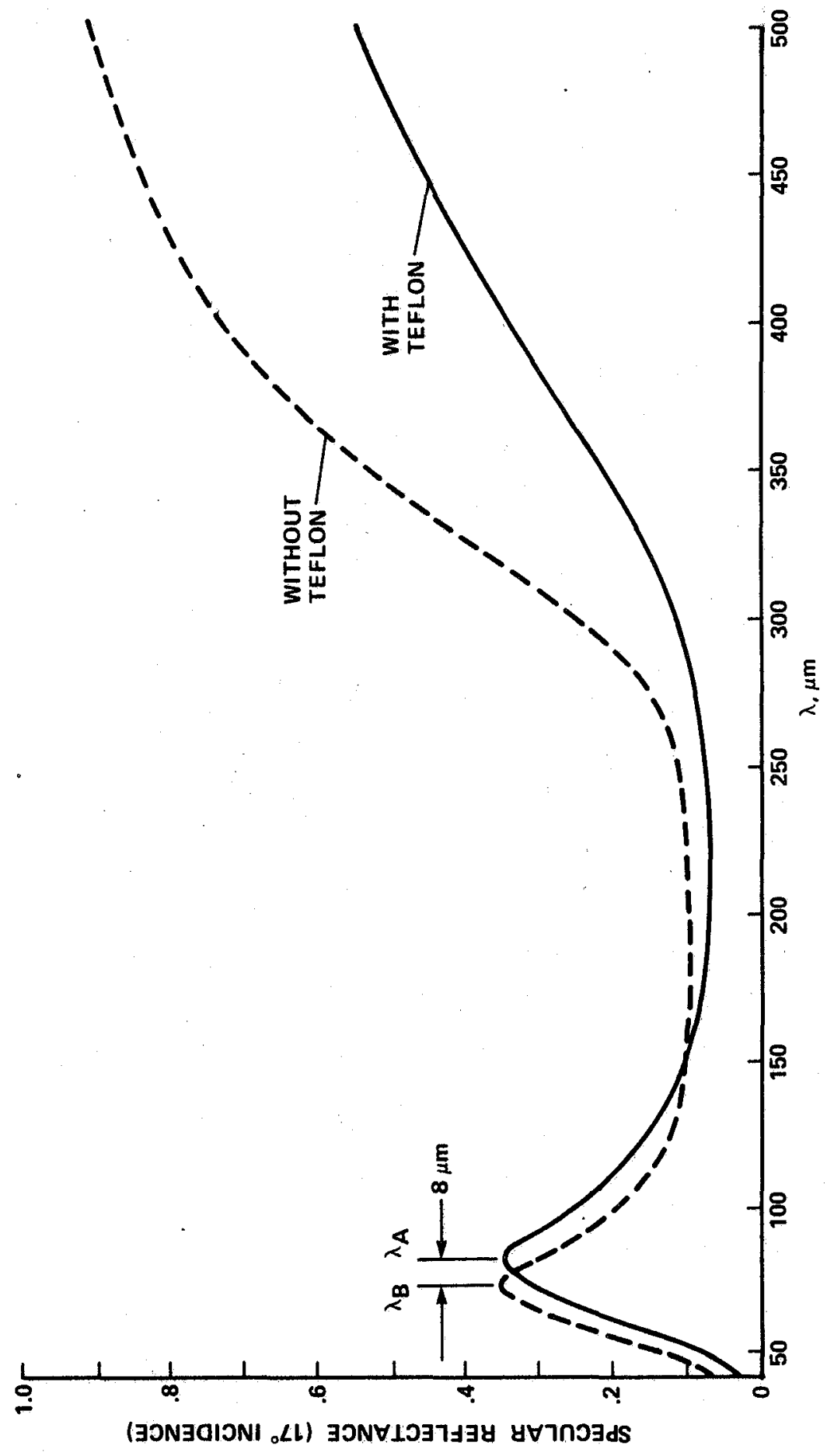

$\underset{+\infty}{+\infty}$ 


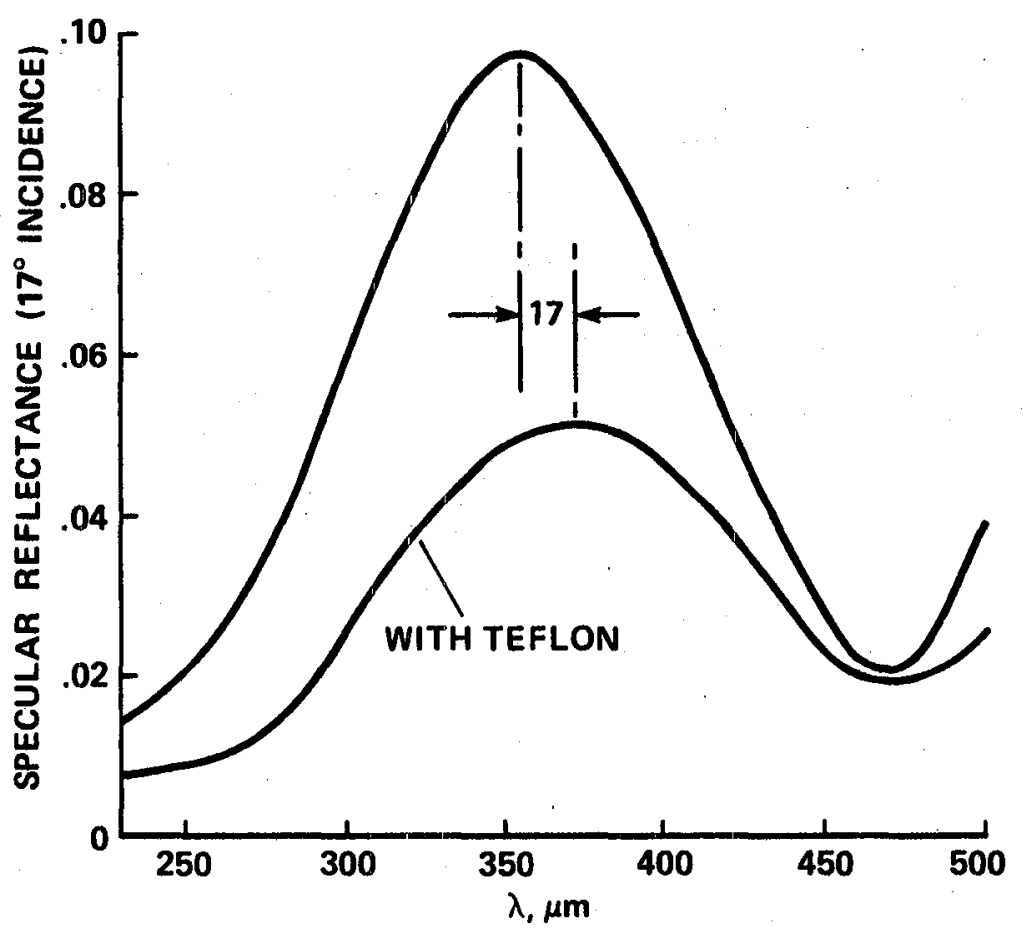

Fig. 2 


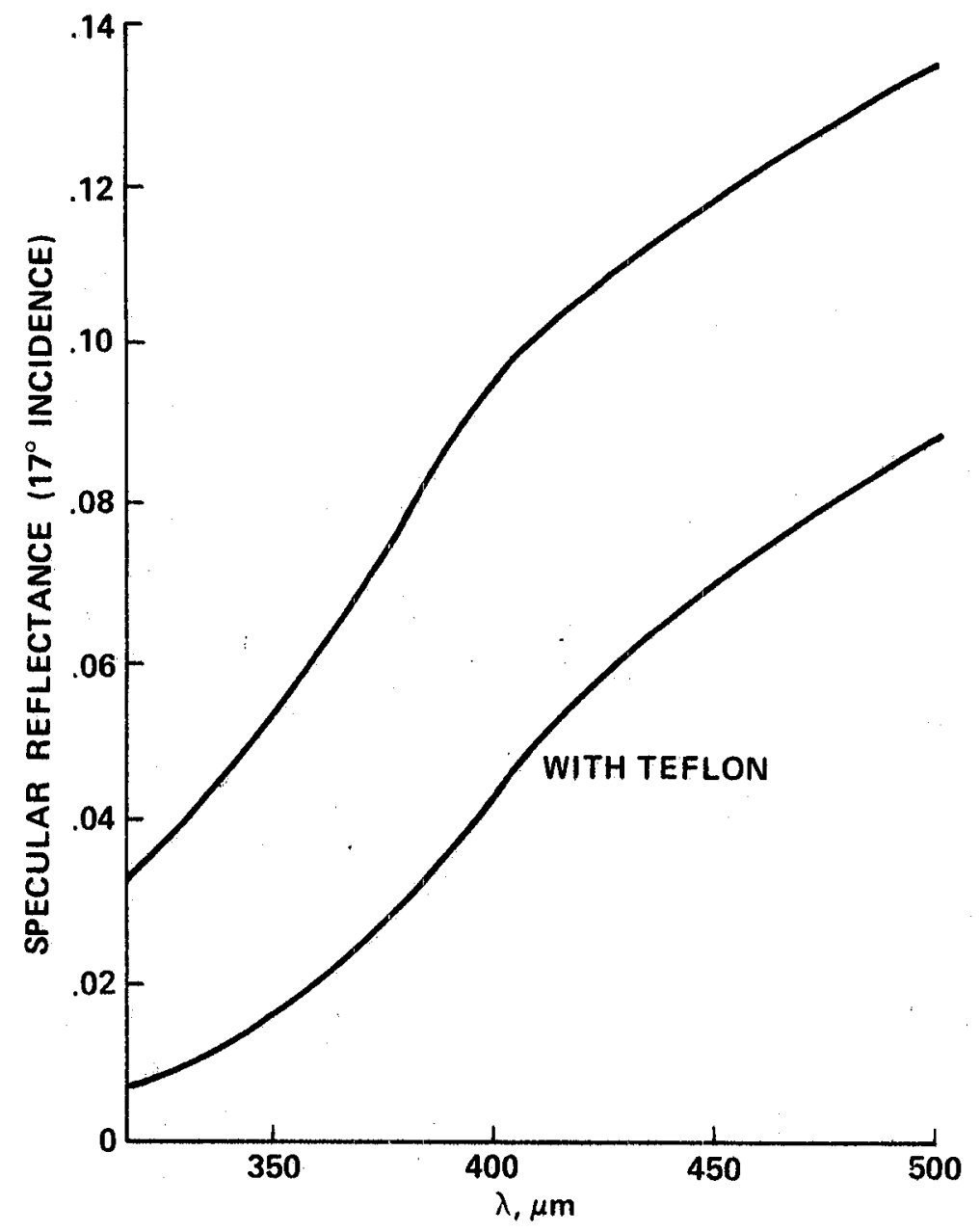

Fig. . 3 


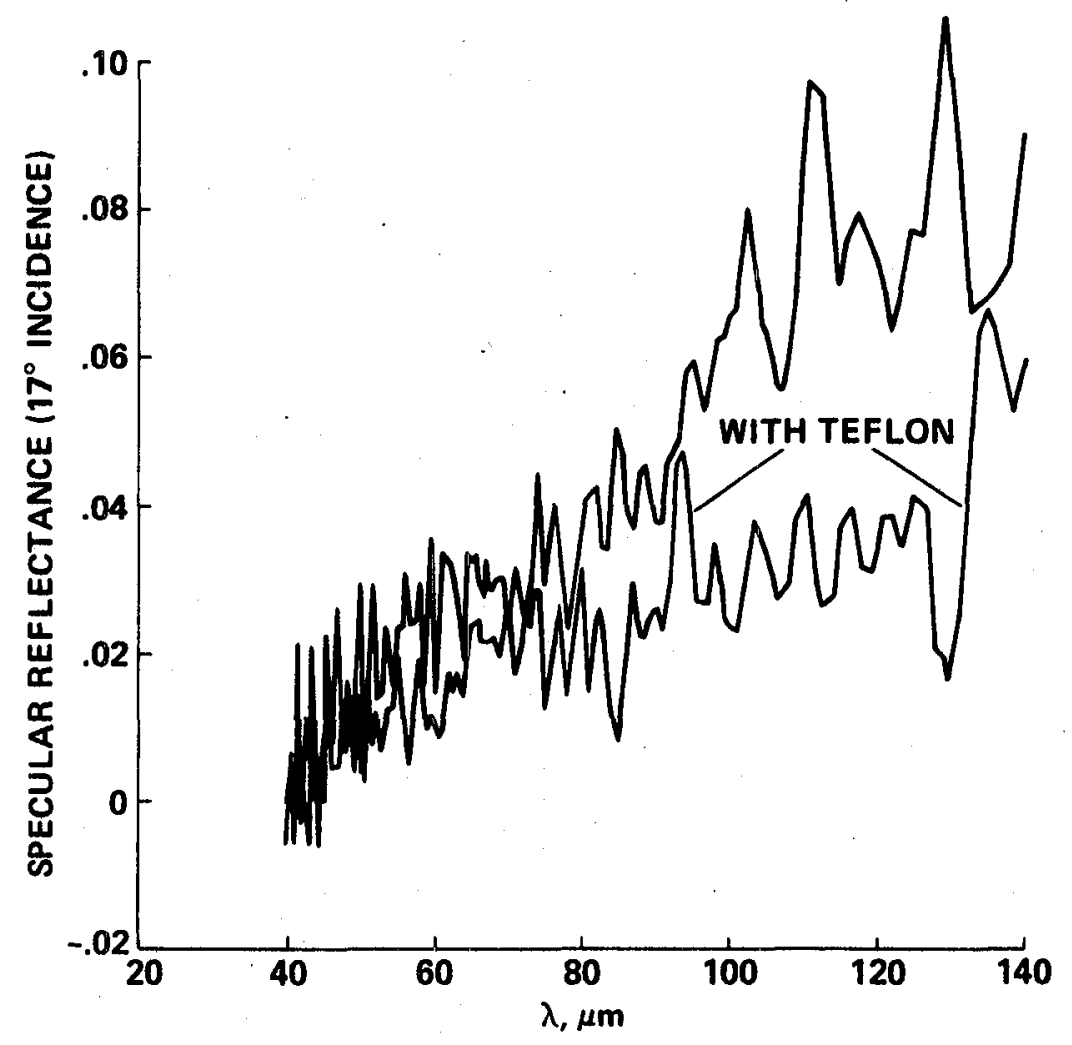

Fig. 4 


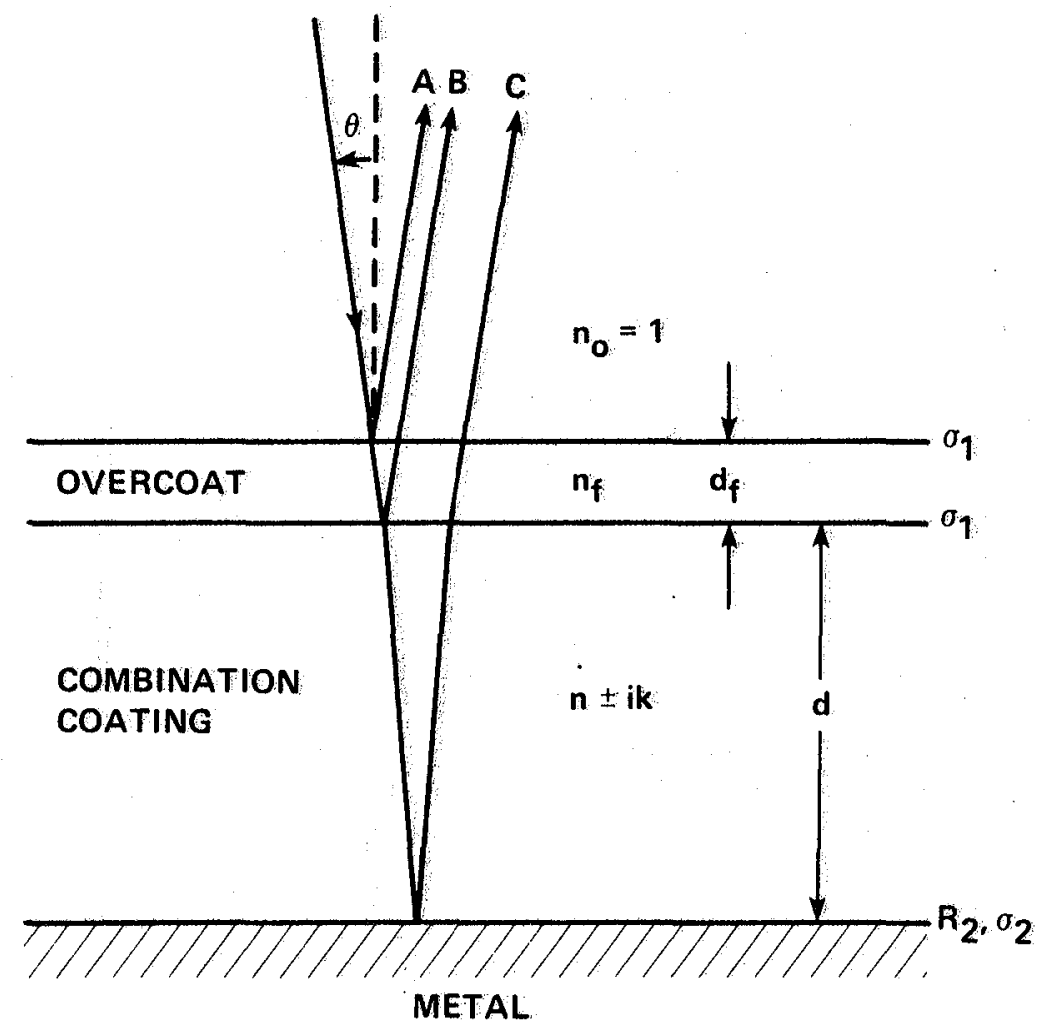

Fig. 5 


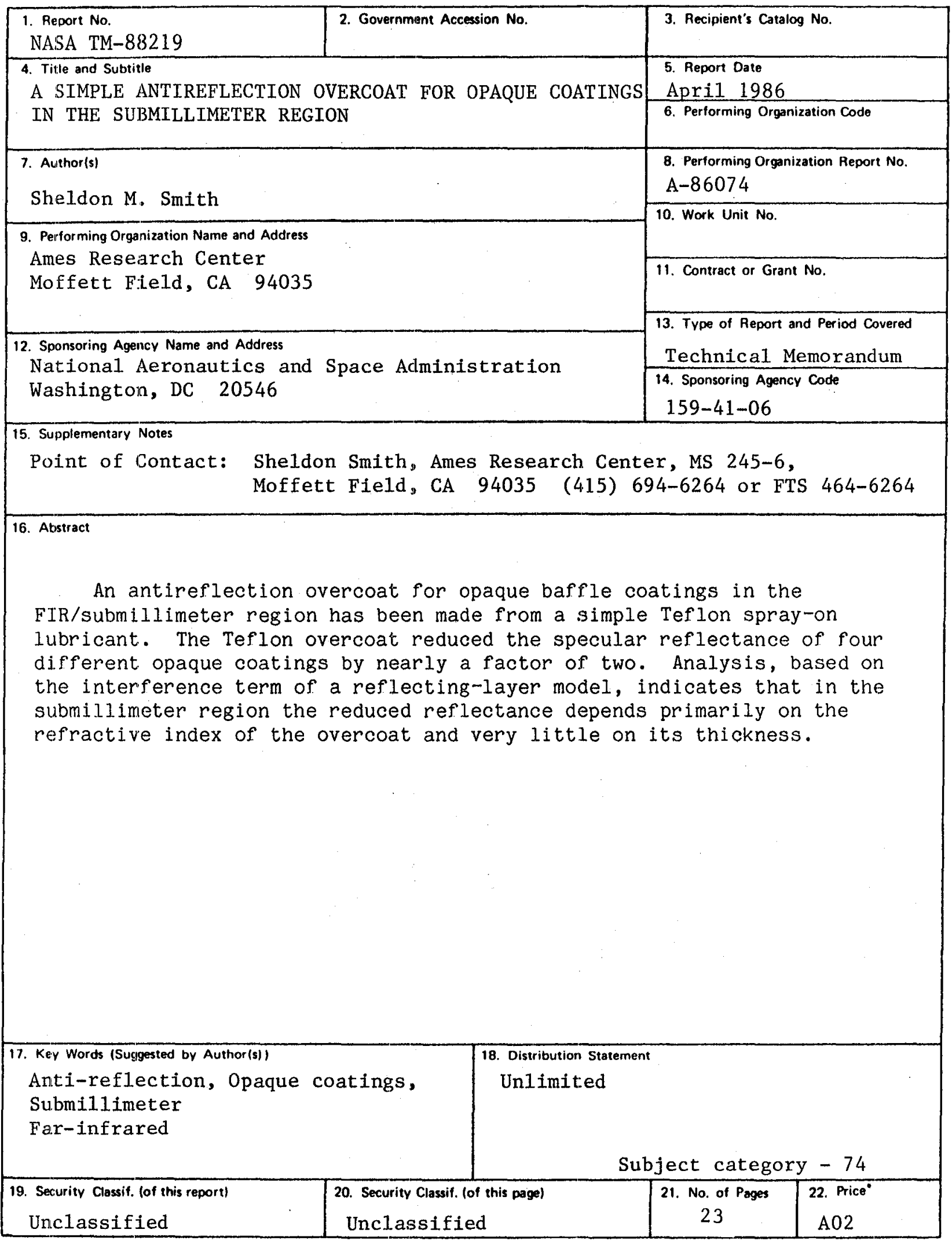

"For sale by the National Technical Information Service, Springfield, Virginia 22161 


\section{End of Document}

